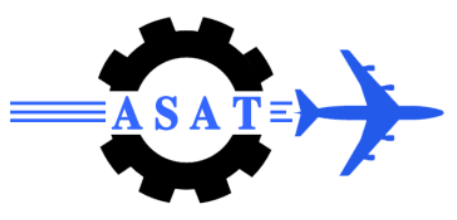

\title{
Using the Swarm Leader Concept for Multi-Agent Obstacle Avoidance
}

\author{
M. Mabrouk
}

\begin{abstract}
The understanding of the operating principles and stability of natural swarms has recently proven to be a useful tool for the design and control of artificial robotic agents. In previous work [1], an algorithm that successfully enhances the navigation performance of a swarm of robots using the swarm leader concept was introduced. An extension to that work is presented in this paper. The real implantation of some applications mentioned in [1] is presented. The new topic in this paper is to use the simulations along with the experimental implementation using a swarming behaviour test-bed, which has been built from scratch to test the swarming behaviour for multi-agent systems. The main objectives are to design and fabricate a mobile robot test-bed, using lego kits as a basis to test the behaviour of 5-6 interacting mobile robots, and to develop a model to simulate the mobile robots' behaviour. These objectives help in designing a series of experiments to explore collective behaviour in swarm robotics with non-complicated way of communication between swarm members. An arena has been installed to perform a range of experiments with the swarm. Through the use of signalling between the agents, such experiments can be developed to achieve high-level goals such as cooperative search tasks and assembly. Experimental and simulation results match in a way that confirms the efficiency of the algorithm as well as its applicability.
\end{abstract}

Keywords: robotics, path-planning, artificial potential field

\section{Nomenclature}

$\begin{array}{ll}C_{a i} & \text { Amplitude of attraction potential of the } \mathrm{i}^{\text {th }} \text { agent } \\ C_{g i} & \text { Amplitude of goal total potential affecting the } \mathrm{i}^{\text {th }} \text { agent } \\ C_{o i} & \text { Amplitude of obstacle potential affecting } \mathrm{i}^{\text {th }} \text { agent } \\ C_{r i} & \text { Amplitude of repulsive potential of the } \mathrm{i}^{\text {th }} \text { agent } \\ l_{a i} & \text { Range of attractive potential for the } \mathrm{i}^{\text {th }} \text { agent } \\ l_{g i} & \text { Range of goal potential affecting the } \mathrm{i}^{\text {th }} \text { agent } \\ l_{o i} & \text { Range of obstacle potential affecting the } \mathrm{i}^{\text {th }} \text { agent } \\ l_{r i} & \text { Range of repulsive potential of the } \mathrm{i}^{\text {th }} \text { agent } \\ m_{i} & \text { Mass of the } \mathrm{i}^{\text {th }} \text { agent } \\ N_{p} & \text { Number of agents } \\ \mathrm{r}_{i g} & \text { Goal }-\mathrm{i}^{\text {th }} \text { agent position vector } \\ \mathrm{r}_{i o} & \text { Obstacle }-\mathrm{i}^{\text {th }} \text { agent position vector } \\ \mathrm{r}_{c} & \text { Swarm center position } \\ V_{g l o b a l}\left(\mathrm{r}_{i}\right) & \text { Global potential field }\end{array}$

\footnotetext{
*Egyptian Armed Forces, Egypt. mhmabrouk2003@yahoo.com
} 


$\begin{array}{ll}V_{\text {goal }}\left(\mathrm{r}_{g}\right) & \text { Goal potential field } \\ V_{\text {interaction }}\left(\mathrm{r}_{i}\right) & \mathrm{i}^{\text {th }} \text { agent interaction potential } \\ V_{\text {obstacles }}\left(\mathrm{r}_{i o}\right) & \text { Repulsive potential of the } N_{o} \text { obstacles } \\ \mathrm{v}_{i} & \mathrm{i}^{\text {th }} \text { agent velocity vector } \\ \beta & \text { Friction self-decelerating force coefficient }\end{array}$

\section{Introduction}

Research activity on autonomous robots has witnessed a surge, especially in the field of artificial robotic systems that inspire ideas from real biological systems due to their important commercial applications [2]. Swarms of self-organizing agents that exchange information have a greater functionality than single robots [3]. In these systems, large numbers of identical autonomous robots are controlled using architectures that are inspired from natural systems such as insect swarms, bird flocks and fish schools [4-7].

In this paper, a model designed to simulate the motion of a swarm of robots, which consists of $N$ agents, is used. The $i^{\text {th }}$ agent is represented with mass $m_{i}$, position $\mathrm{r}_{i}$ and relative distance $\mathrm{r}_{i j}$ between the $i^{\text {th }}$ and $j^{\text {th }}$ agents. The generalized Morse potential, which decays exponentially at large distances and represents a comparatively realistic description of natural swarming agents, is used to define the social potential interactions amongst the swarm agents $V_{\text {interaction }}\left(\mathrm{r}_{i}\right)$, the attraction potential of the goal $V_{\text {goal }}\left(\mathrm{r}_{i g}\right)$ and the repulsive potential of the $N_{o}$ obstacles $V_{\text {obstacles }}\left(\mathrm{r}_{i o}\right)$. Unit mass agents are considered for simplicity. To prevent the agents from reaching large speeds, a dissipative frictional force with coefficient $\beta_{i}$ corresponding to the $i^{\text {th }}$ agent is added. The potential is characterized by attractive and repulsive interaction potential fields of strength $C_{a}$ and $C_{r}$ with ranges $l_{a}$ and $l_{r}$ respectively, while $C_{g}, l_{g}, C_{o z}$ and $l_{o z}$ are the attraction potential strength and range of the goal point and the repulsive potential strength and range of the $z^{\text {th }}$ obstacle point, respectively. The equations of motion for $N$ agents that contains $N_{o}$ obstacle points and a goal point at position $G$, are then defined for the $i^{\text {th }}$ agent as [8]:

$\mathbf{v}_{i}=\dot{\mathbf{r}}_{i}$

$m_{i} \dot{\mathbf{v}}_{i}=-\beta_{i} \mathbf{v}_{i}-\nabla_{i} V_{\text {global }}\left(\mathbf{r}_{i}\right)$

$V_{\text {global }}\left(\mathbf{r}_{i}\right)=V_{\text {interaction }}\left(\mathbf{r}_{i}\right)+V_{\text {goal }}\left(\mathbf{r}_{i g}\right)+V_{\text {obstacles }}\left(\mathbf{r}_{i o}\right)$

$$
\begin{aligned}
V_{\text {global }}\left(\mathbf{r}_{i}\right)= & \sum_{j \neq i}^{N}\left(C_{r_{j}} e^{-\left|\mathbf{r}_{i}-\mathbf{r}_{j}\right| / l_{r_{j}}}-C_{a_{j}} e^{-\left|\mathbf{r}_{i}-\mathbf{r}_{j}\right| / l_{a_{j}}}\right) \\
& -C_{g} e^{-\left|\mathbf{r}_{i}-\mathbf{r}_{g}\right| / l_{g}}+\sum_{z=1}^{N_{o}} C_{o_{z}} e^{-\left|\mathbf{r}_{i}-\mathbf{r}_{o_{z}}\right| / l_{o_{z}}}
\end{aligned}
$$

In earlier work [8], sets of first order differential equations to describe the free parameters of the potential field (internal state) to solve the local minimum problem were introduced. For artificial potential field based navigation, there have been several attempts to solve the local 
minimum problem. The problem for a swarm of agents attracted to a goal point at position $\boldsymbol{G}$ is defined such that an artificial potential field at $\boldsymbol{G}$ induces motion towards the goal. However, in order to prevent collision with a static obstacle, an additional repulsive potential field is required. In general, a local minimum may form due to the superposition of the goal potential and that of the obstacles, resulting in the agent, or swarm of agents, becoming trapped in a state other than the goal $\boldsymbol{G}$. Considering this problem, the entire swarm, or part of the swarm will be trapped at the obstacle since the agents trapped inside the obstacle will experience two virtual opposite forces, as shown in Fig. 1.

Three new concepts were introduced to solve the problem for a swarm of robots; the swarm leader concept [9], the swarm aggregation concept [10], and the swarm vortex-like behaviour concept [11] to enhance the performance of the internal state model for agents' behaviour that allows them to effectively solve this key problem. In this paper, some applications are introduced using the internal state model enhanced with the swarm leader concept through simulation and test-bed facility. The two core points of this paper are (1) the construction of the test-bed facility explained in section 4, and (2) the experimental results obtained from real implementation of a swarm of robots navigation using the test-bed facility.

\section{Agent Internal State Model with Swarm Leader Concept}

There is a considerable body of research concerning building artificial systems inspired by swarm leaders phenomenon in real biological systems. In [12, 13], the authors introduced dynamic models based on the distance and the angle between leaders and followers, which indicates that the agents must know who and where their leaders are. Different leader roles were discussed in [14] and a convergent condition, in which the followers need to have the leaders' states by sharing global information, was constructed by using contraction theory. In [15], the swarm members know which members are the leaders in a leader based control strategy. Similarly, the followers need to know who the leaders are in [16] where the leaderfollower systems are investigated in terms of controllability and optimal control. In [17], the authors discussed the importance of updating the follower information concerning the leader position. Experimentally, few research work focuses on leader-follower relationship in multiagent systems. McLurkin [3] tested a set of communication techniques and a library of behaviors, among which follow-the-leader behaviour is programmed, on a swarm of 100 physical robots.

From this background it can be seen that although local information can be used to control the relative distances and angles amongst swarm members, the followers must know which members are their leaders. In addition, the followers need to share global information about the updated position of their leader. In the algorithm used in this paper, the agents have information about the leaders by sharing global information, which are the agents' potential parameters that are employed to express the leader-follower relationship [9]. The swarm leader concept enables the swarm in Fig. 1 to efficiently solve the problem by making the agents follow the agent that finds a clear way to the goal, as shown in Fig. 2.

\section{Attraction of Agents to a Temporary Leader}

For a complete understanding of the role of the leader in a free system and how it may affect the behaviour of swarm robots using the artificial potential approach for the agents interactions, the motion of a free swarm point robots whose agents experience an attraction to one of them will be considered. For a swarm of agents, let agent $(\boldsymbol{h})$ is the temporary leader that has higher $\boldsymbol{C}_{\boldsymbol{a}}$ and $\boldsymbol{l}_{\boldsymbol{a}}$ than the other agents of the swarm. Recalling Eq. (1-4) for obstacles and goal free environment and using $\boldsymbol{V}_{\boldsymbol{i}}$ instead of $\boldsymbol{V}_{\text {interaction }}$ for simplicity, the global potential equation for $i^{\text {th }}$ agent is 
$V_{\text {global }}\left(\mathbf{r}_{i}\right)=V_{i j}\left(\mathbf{r}_{i}\right)=\sum_{j \neq i}^{N_{p}}\left(C_{r_{j}} e^{-\left|\mathbf{r}_{i}-\mathbf{r}_{j}\right| / l_{r_{j}}}-C_{a_{j}} e^{-\left|\mathbf{r}_{i}-\mathbf{r}_{j}\right| / l_{a_{j}}}\right)$

Defining the swarm center position as $\mathbf{r}_{c}=\frac{1}{N_{p}} \sum_{i=1}^{N_{p}} \mathbf{r}_{i}$

and noting that $\nabla_{i} V_{i h}\left(\mathbf{r}_{i h}\right)=V_{i h}^{\prime}\left(\mathbf{r}_{i h}\right) \hat{\mathbf{r}}_{i h}$, the equation of motion of the swarm center will be

$\ddot{\mathbf{r}}_{c}=-\frac{1}{N_{p}}\left(\sum_{i=1}^{N_{p}} \beta \mathbf{v}_{i}+\sum_{i=1}^{N_{p}} \sum_{\substack{j \neq i \\ i \neq h \\ j \neq h}}^{N_{p}} \nabla_{i} V_{i j}+\sum_{i=1}^{N_{p}} V_{i h}^{\prime}\left(\mathbf{r}_{i h}\right) . \hat{\mathbf{r}}_{i h}\right)$

Since $\hat{\mathbf{r}}_{i j}=-\hat{\mathbf{r}}_{j i}$, the double summation in Eq. (6) will cancel to yield

$\ddot{\mathbf{r}}_{c}+\frac{\beta}{N_{p}} \dot{\mathbf{r}}_{c}=-\frac{1}{N_{p}} \sum_{i=1}^{N_{p}} V_{i h}^{\prime}\left(\mathbf{r}_{i h}\right) \cdot \hat{\mathbf{r}}_{i h}$

which represents a damped oscillator with a forcing term generated by the lead agent [18]. It can therefore be concluded that the social potential field can be used in a way that the agents will be attracted to any single agent considered as a temporary goal if it has a larger attraction interaction parameter according to some task; for example a scout agent finding something of interest for the rest of the swarm.

\section{Simulation Results}

To show the swarm leader concept, Eq. (1) - Eq. (4) are now used to simulate the agents' motion for $N_{p}$ identical agents which are trapped behind a barrier that consists of $N_{o}$ identical obstacle points, as shown in Fig. 3, where $\boldsymbol{G}$ is a goal point which has an attraction potential of low interaction range $l_{g}$ such that it does not extend to the region inside the trap. The challenge in this situation is that the agents are stuck inside a trap whose only exit is located away from the goal position and they are not strongly attracted to the goal. In this situation, a condition is defined such that if one of the agents finds its way through the exit, it will gain a higher attraction potential coefficient $\boldsymbol{C}_{\boldsymbol{a}}$ and higher attraction potential range $\boldsymbol{l}_{\boldsymbol{a}}$. These conditions now make any succeeding agent a temporary leader for the rest of the agents and the swarm center therefore accelerates to the leader position, leading them out of the trap. Then, the leader and subsequently the swarm are attracted to the goal. Figure 3 shows the agents randomly moving inside the trap, as shown in Fig. 3.a), until one agent succeeds in escaping, as shown in Fig. 3.b) and then becomes a temporary leader for the rest of the agents, as shown in Fig. 3.c) leading them out of the trap, as shown in Fig. 3.d) until the group members reach the goal point, as shown in Fig. 3.e-f). 


\section{Experimental Setup}

The escaping the trap application has been implemented in a swarming behaviour test-bed, the basic contents of this test-bed will be briefly discussed in the following sub-section.

\subsection{Swarm Robotics Test-Bed}

The implementation of the experiment requires that the used test-bed is properly equipped to satisfy the requirements for the simulation of local minimum defined by [19], and obstacle avoidance problem solving techniques be carried out utilizing Lego ${ }^{\circledR}$ Mindstorms autonomous robotic agents for simplicity. The development of the test bed entailed three basic parts the agents, the arena and Computer software.

The room where the test-bed should be located required special preparations before it could be used for an effective test bed session. Since the robotic agents would be responding to light sources, and the sensory equipment which react to this stimulus are very sensitive, it is very important that the lab room be completely darkened. Also the floor of the arena should be smooth plane for the agents to manoeuvre around, as a loss of traction may occur due to uneven ground.

An overview of the test bed facility that has been built to test the swarming behaviour for multi-agent systems is shown in Fig. 4. The arena perimeter served to define the workspace in which the agents would inhabit. It is simply manufactured by joining four $5.5 \mathrm{~mm}$ thick plywood planks together at their ends using masking tape. The arena perimeter measures $2.4 \mathrm{~m}$ by $2.4 \mathrm{~m}$, and the planks are cut $100 \mathrm{~mm}$ wide, making the perimeter stand the same height as the $\mathrm{C}$-shaped barrier. As the local minimum problem regarding a $\mathrm{C}$-shaped barrier is to be examined, a suitable barrier has been manufactured. The used C-shaped Perspex barrier satisfies the requirements for the simulation of local minimum defined by [19] which are it must be completely transparent as the agents should be attracted to the light source on the other side of the barrier without detecting the obstacle until reaching "about to touch" it.

A standard desk lamp is used as the goal position. As the agents react to very small changes in light intensity, it is important that the light could be distributed uniformly along the ground around the source. This uniform distribution is ensured by using a 40-Watt reflector bulb in the lamp. The lamp is then suspended directly above the area on the ground acting as the goal position. Having the lamp suspended over the arena boundary ensured that nothing except the C-shaped barrier would interfere with the agent's progress.

In order to utilize an appropriate tracking facility, it is required that a suitable camera be suspended over the arena. After examining the capabilities of the robots user interaction software, it is decided that a standard web cam in addition to a night vision camera are suitable to make good documentation and afterward analysis of the results. Both cameras are connected via standard USB wire connection to a computer and suspended above the arena.

When talking about the agents, every agent includes an NXT Intelligent Brick with 32-bit microprocessor, 3 Interactive Servo motors, an improved touch sensor, and an improved light sensor. The test-bed has been built to test the behaviour of identical agents, which interact via pair-wise interactions of short-range repulsions and long-range attraction. Every leader agent, as shown in Fig. 5, is modified by equipping it with an external source of light, a short-range touch sensor to implement the repulsion type forces, and long-range light sensors to implement the attraction type forces. The same procedures in the rescue mission application are implemented using only 3 agents for simplicity. 


\subsection{Experimental Results}

The scenario of the local minimum problem, proposed in [19], is implemented using an agent that is attracted to a visible goal (light source located at the goal position) through a transparent barrier. When the agent reaches the barrier, it is repelled according to the function of its touch sensor. However, as the agent moves away from the barrier it is attracted again according to the function of its light sensor and then tries again to reach the goal through the barrier, as shown in Fig. 6.a). In this case, the agent gets stuck in a position away from the goal position defining the local minimum problem. In the simulation, using the internal states model enables the stuck agent to solve the problem by detecting the location of another agent 'the leader' that has a clear path to the goal as shown in Fig. 6.b) then following it.

This idea is implemented through a modification of the leader agent, shown in Fig. 5, such that it gains leader properties (the external light source becomes brighter which represents for higher $\boldsymbol{C}_{\boldsymbol{a}}$ according to the internal state model with the swarm leader concept) as it has a clear way to the goal. This enables the leader agents to attract the trapped agent more than the goal such that the individual trapped in the local minimum is attracted to the leaders rather than to the goal, as shown in Fig. 6.c) then maneuvering successfully around the barrier as shown if Fig. 6.d-e) until reaching the goal as shown in Fig. 6.f).

\section{Conclusions}

This paper presents the real implementation of an application which is based on earlier work [1], which aimed to enhance the artificial potential fields (APF) based navigation performance of multi-agent systems using one of the most common swarming behaviour in natural systems; the swarm leader concept. The escaping the trap application is introduced using simulations through which if one of the agents finds its way through the exit, it will gain a higher attraction potential coefficient $\boldsymbol{C}_{\boldsymbol{a}}$ and higher attraction potential range $\boldsymbol{l}_{\boldsymbol{a}}$ becoming a temporarily leader to the rest of the individuals inside the trap to help them escape. A test-bed is built to implement the rescue mission that is introduced by simulation. The setup of the test-bed involves modifying the agents to satisfy swarm leader task, developing the algorithms, and finally assembling the arena itself. Each of these three stages is briefly covered. The implementation of the escaping the trap application using a swarming behaviour test-bed confirms the applicability of the used model as well as its ability to enhance the performance for a real swarm of robots.

\section{References:}

[1] Mabrouk, M., Overcoming Local Minima in Artificial Potential Field Path-Planning Using Internal Agent States, PhD thesis, University of Strathclyde, 2009.

[2] Webb, B., "Can robots make good models of biological behaviour?"," The Behavioural and Brain Sciences" 24 (6), 2003.

[3] McLurkin, J., Stupid Robot Tricks: A Behavior-Based Distributed Algorithm, MSc Thesis. MIT, Library for Programming Swarms of Robots, 2004.

[4] Bonabeau, E., Dorigo, M., and Theraulaz, G., Swarm intelligence: From natural to artificial systems, Oxford University Press, New York, 1999.

[5] Gazi, V., and Passino, K., "Stability analysis of swarms", "IEEE Trans. Autom. Contr. Vol. 48", pages 692-697, 2003.

[6] Nguyen, B. Q., Chuang, Y. L., Tung, D., Hsieh, C., Jin, Z., Shi, L., Marthaler, D., Bertozzi, A. L., and Murray, R. M., "Virtual attractive repulsive potentials for cooperative control of second order dynamic vehicles on the caltech MVWT", "Proc. American Contr. Conf.", pages 1084 -1089. Portland, OR,USA, 2005. 
[7] Chuang, Y. L., Huang, Y. R., D’Orsogna, M. R., and Bertozzi, A. L., "Multi-vehicle flocking: Scalability of cooperative control algorithms using pairwise potential", "IEEE International Conference on Robotics and Automation", 2292-2299, 2007.

[8] Mabrouk, M., and McInnes, C., "Solving the potential field local minimum problem using internal agent states", "Journal of Robotics and Autonomous Systems", 56, 1050_1060, 2008.

[9] Mabrouk, M., and McInnes, C., "Swarm robot social potential fields with internal agent dynamics", "Proceeding of the 12th International Conference on Aerospace Sciences and Aviation Technology (ASAT12)”, ROB-02:1-14. Cairo, Egypt, 2007.

[10] Mabrouk, M., and McInnes, C., "Swarm Potential Fields with Internal Agent States and Collective Behaviour", "Proceeding of Towards Autonomous Robotic Systems, TAROS", pp. 90-96, 2007.

[11] Mabrouk, M., and McInnes, C., "Wall following to escape local minima for swarms of agents using internal states and emergent behaviour", "International Conference on Computational Intelligence and Intelligent Systems, ICCIIS”, Vol.1, pp. 24-31 2008.

[12] Desai, J., Ostrowski, J., and Kumar, V., "Modelling and control of formations of nonholonomic mobile robots", "IEEE transactions on robotics and automation", vol.17, No.6, pp.905-908, 2001.

[13] Das, A., Fierro, R., Kumar, V., Ostrowski, J, Spletzer, J. and Taylor, C., "A visionbased formation control framework", "IEEE transactions on robotics and automation", vol.18, No.5, pp.813-825, 2002.

[14] Wang, W., and Slotine, J., "A theoretical study of different leader roles in networks", "IEEE transactions on robotics and automation", vol.51, No.7, pp.1156-1161, 2006.

[15] Dimarogonas, D., Egerstedt, M. and Kyriakopoulos, K., "A leader-based containment control strategy for multiple unicycles", "Proceedings of the IEEE conference on decision and control", pp. 5968-5973, 2006.

[16] Ji M., Muhammad A., and Egerstedt M., "Leader-based multi-agent coordination: controllability and optimal control", "Proceedings of the American control conference", 2006.

[17] Wang, Z. and Gu, D., "Fuzzy control of leader-follower flocking system", "Proceedings of towards autonomous robotic systems", TAROS 2007, pp 70-77, 2007.

[18] Beer P., Johnston E. and Clausen W. Vectors mechanics for engineers: Dynamics. McGraw-Hill Higher Education. ISBN 978-007-127360-2, 2007.

[19] Balkenius, C. "Natural Intelligence for Autonomous Agents", (extended version), Lund University Cognitive Studies, 29,1994. 


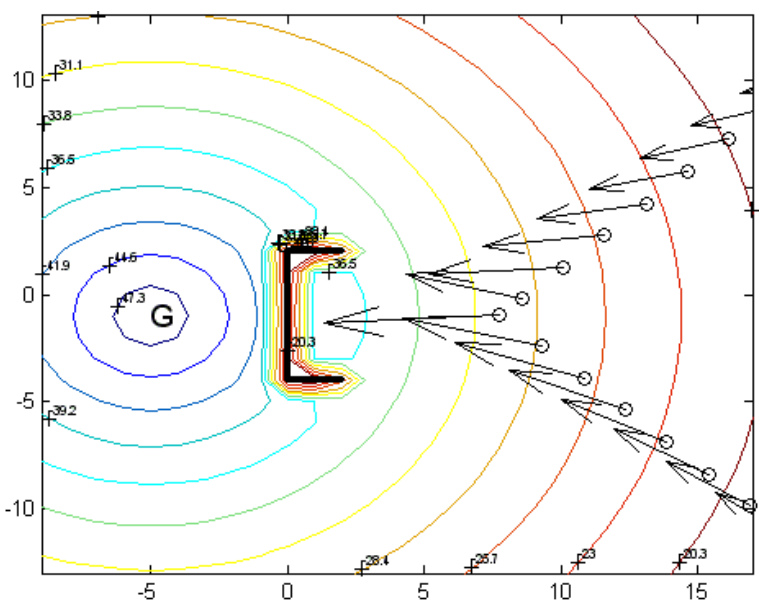

(a) $t=1$

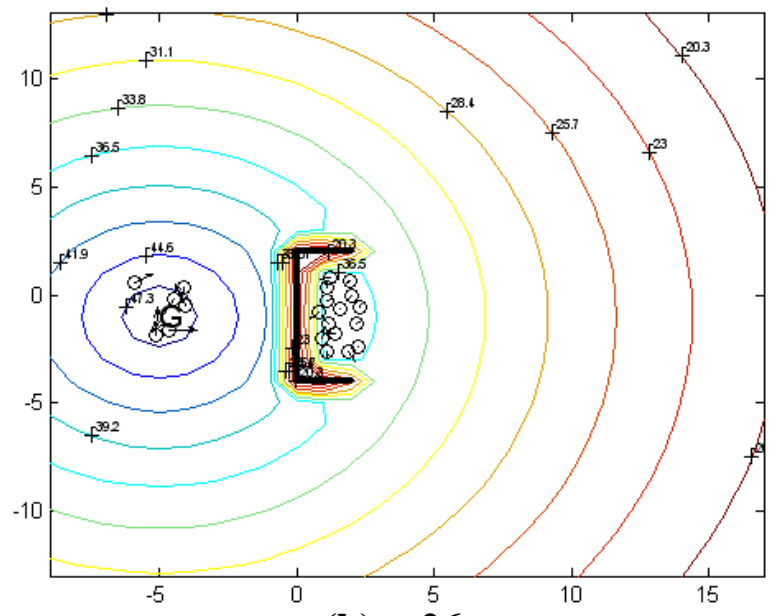

(b) $t=26$

Fig. 1 Classical reactive problem for a swarm of agents 


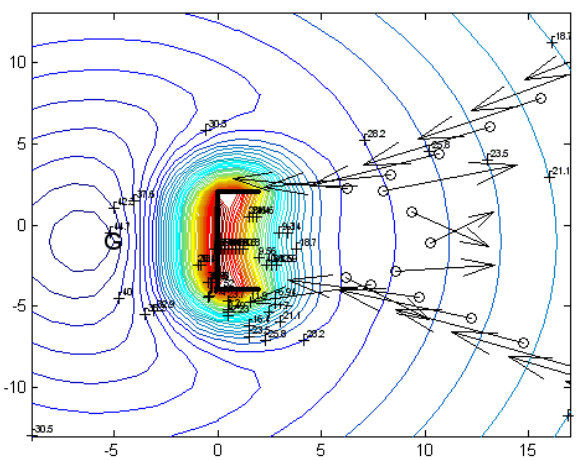

(a) $t=12$

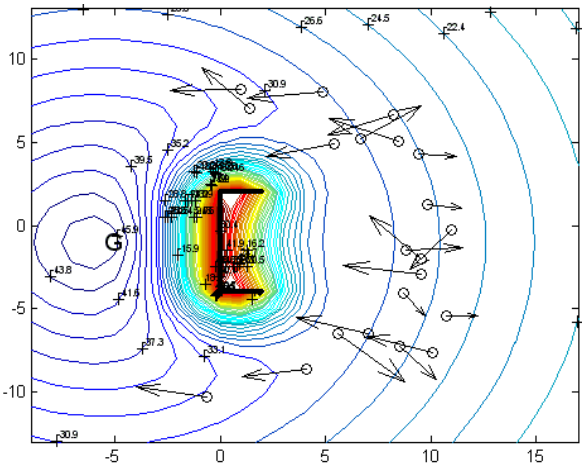

(b) $t=24$

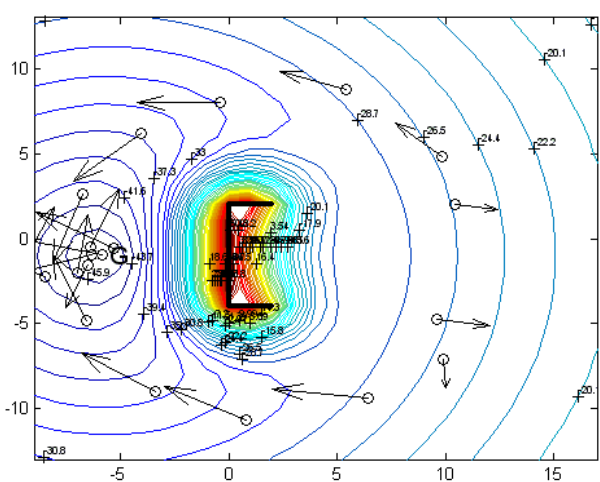

(c) $t=48$

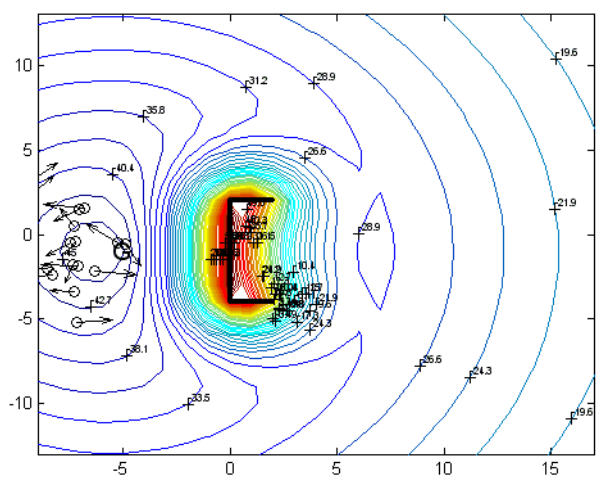

(d) $t=107$

Fig. 2 Behaviour of a swarm using the internal state model and swarm leader, $t=12-107$, [9] 


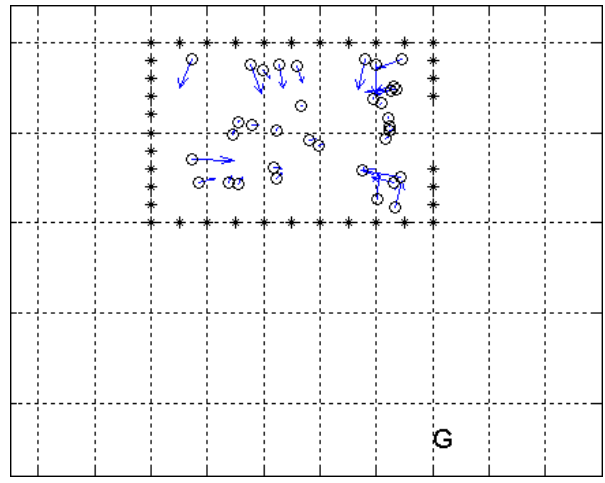

(a) $t=0.5$

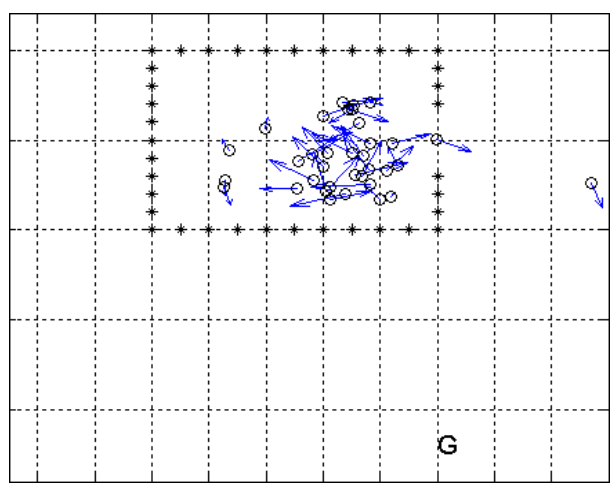

(c) $t=12$

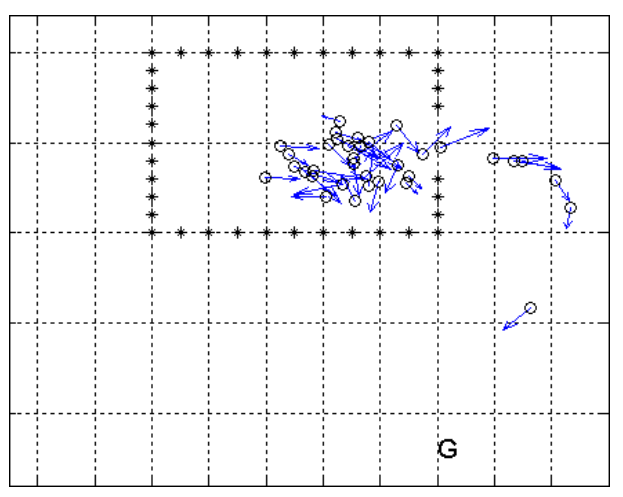

(e) $t=18$

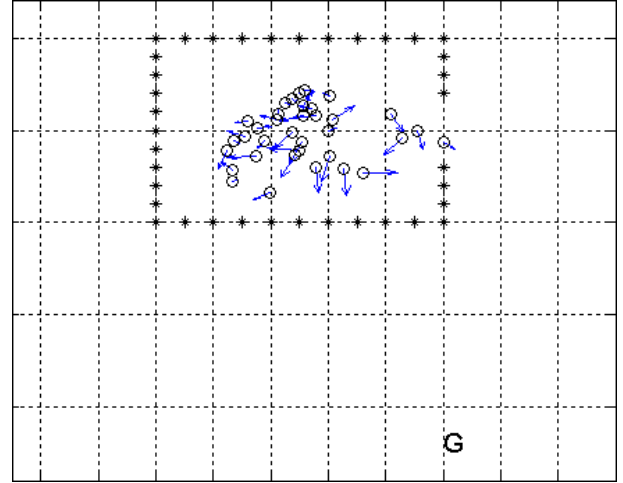

(b) $t=9$

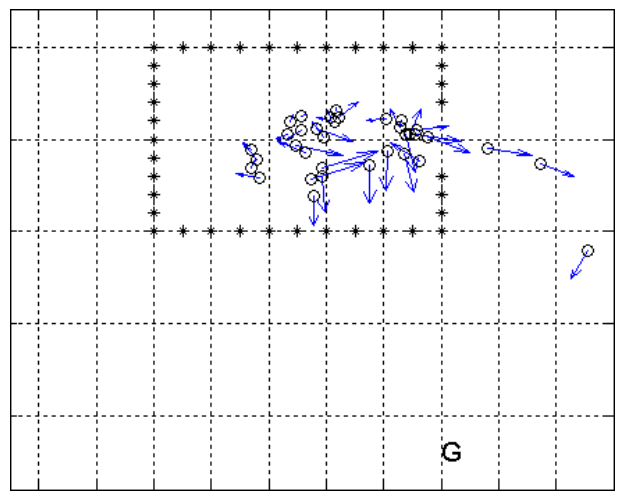

(d) $t=15$

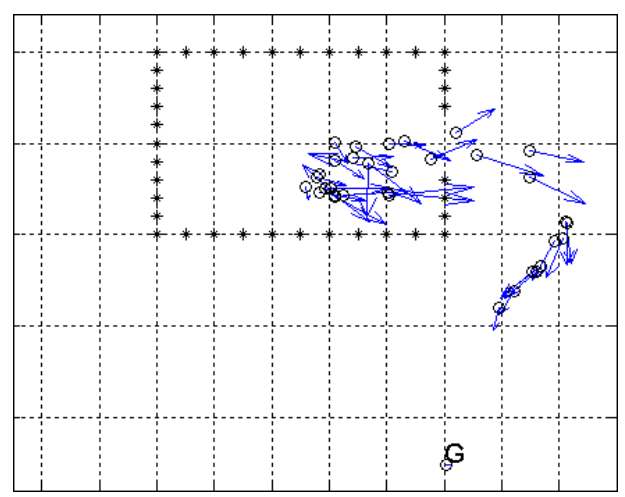

(f) $t=23$

Fig. 3 Swarm leader concept in a trap application 

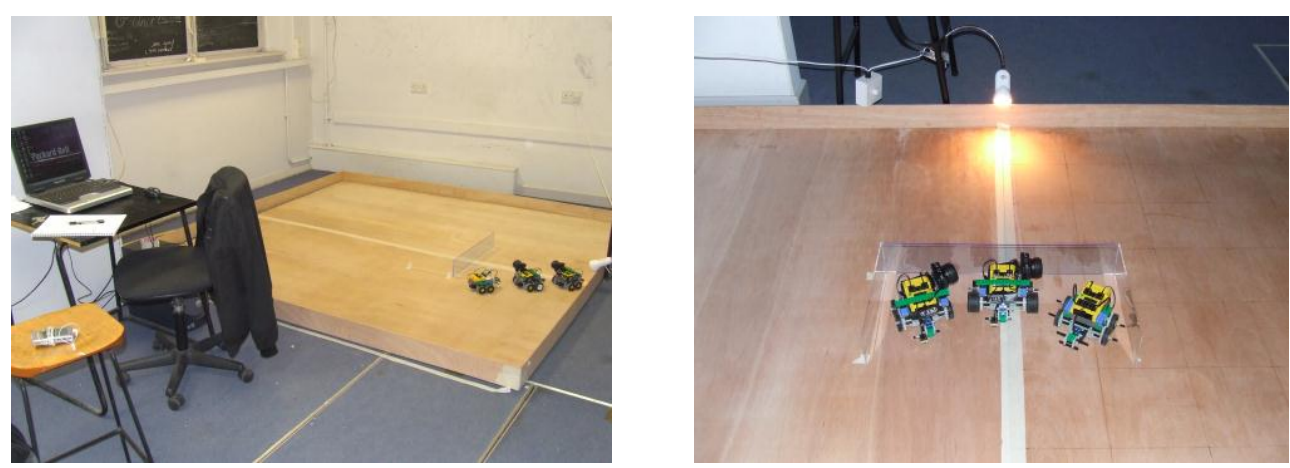

Fig. 4 Global overview of the swarm robotics test-bed

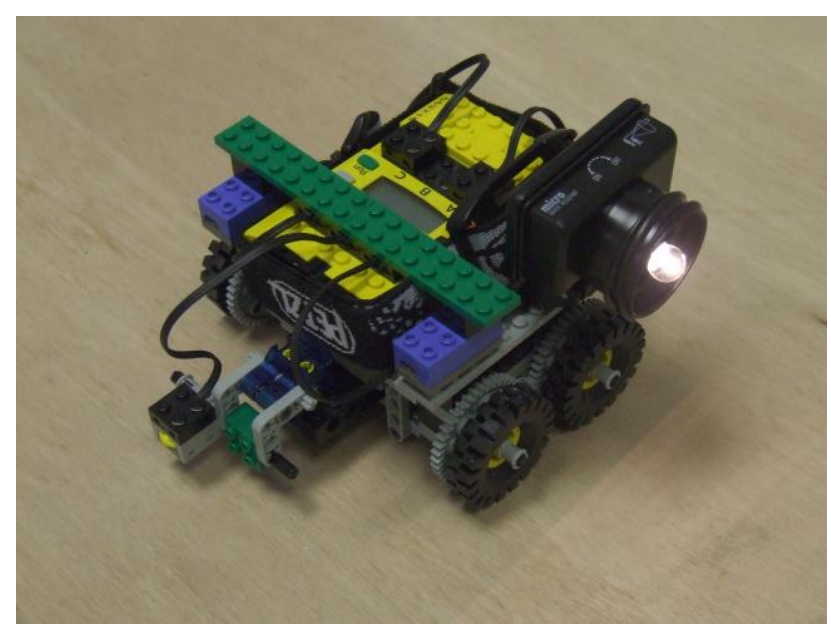

Fig. 5 One of the leader agents used in the test 


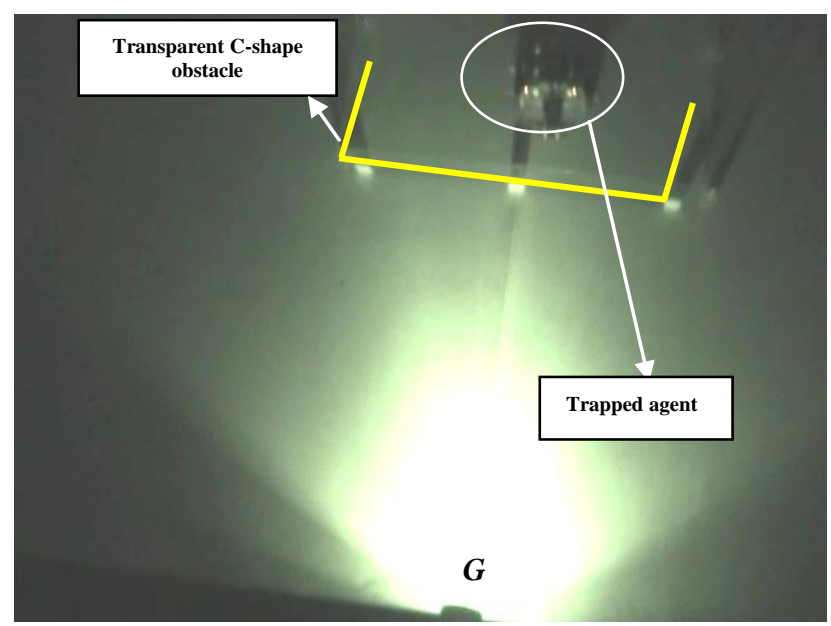

(a) $t=1$

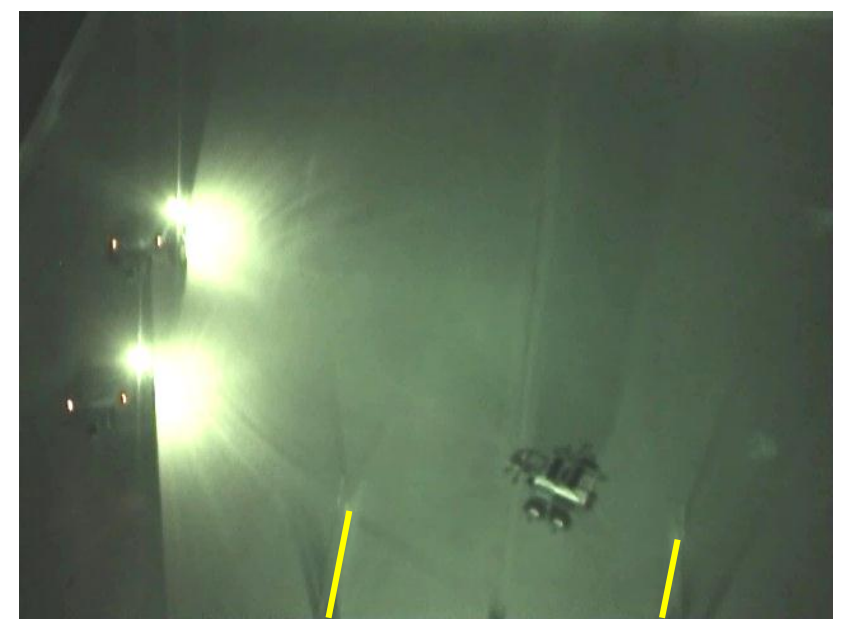

(c) $t=18$

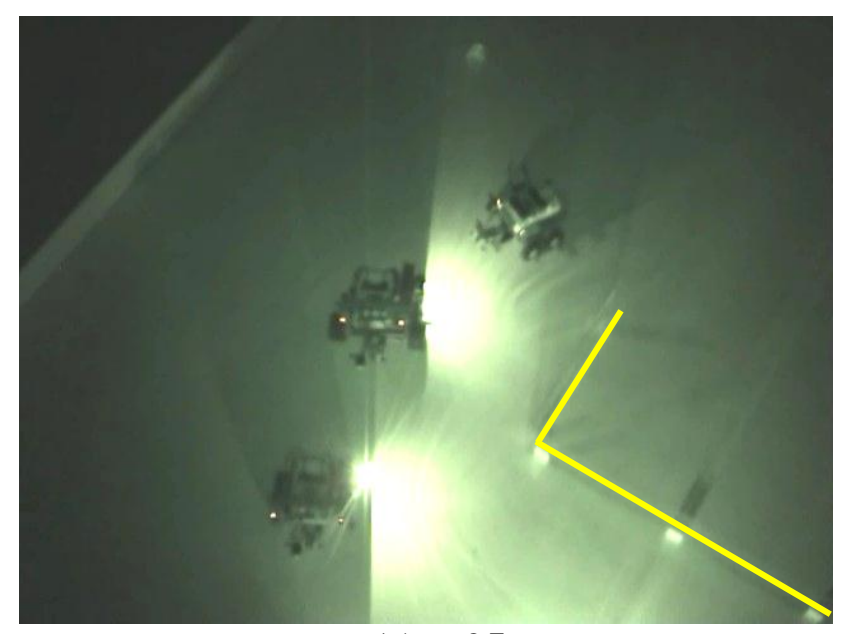

(e) $t=25$

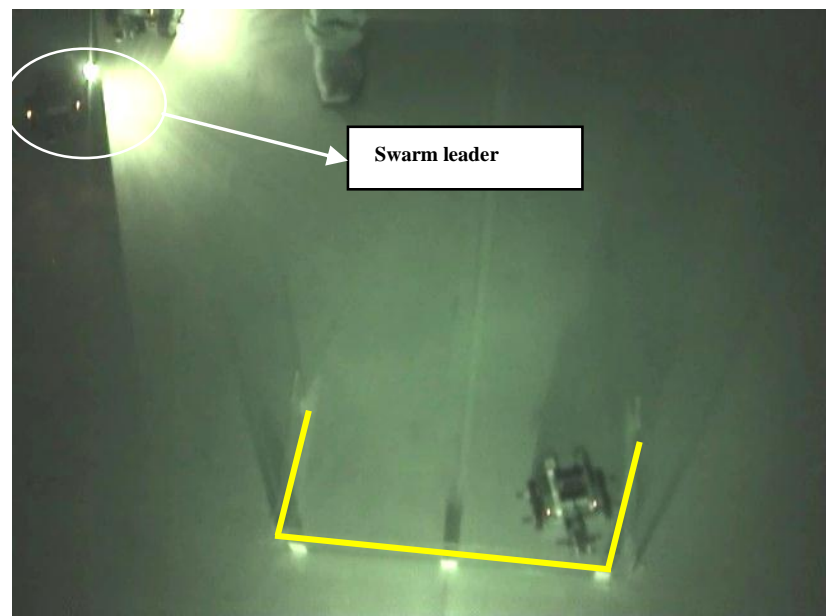

(b) $t=10$

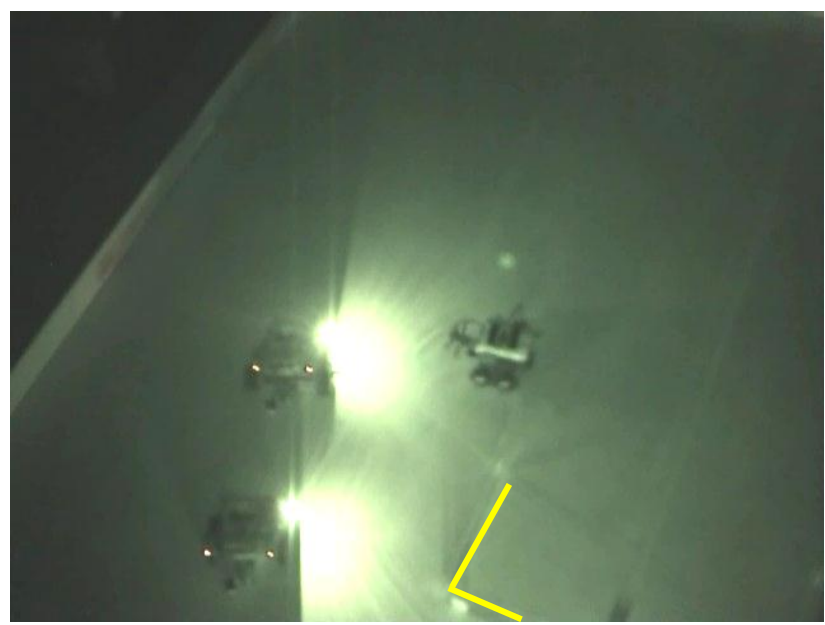

(d) $t=21$

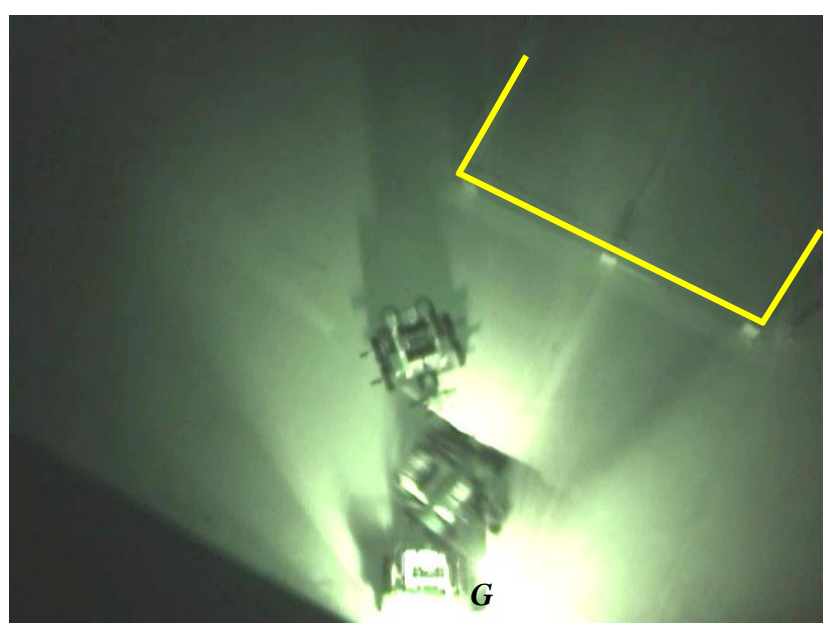

(f) $t=36$

Fig. 6 Swarm leader concept implementation 